\title{
Finite-SNR Diversity-Multiplexing Tradeoff for Cooperative Transmissions with Opportunistic Network Coding
}

\author{
Guoyou Li, Yajian Zhou, Jingxian Zhou, \\ Kangfeng Zheng, and Yixian Yang \\ Information Security Center, Beijing University of Posts and Telecommunications, P.O. Box 145, \\ Beijing 100876, China \\ Correspondence should be addressed to Guoyou Li, lgyoudavid@126.com
}

Received 1 October 2012; Accepted 29 November 2012

Academic Editor: Ming Li

Copyright (c) 2012 Guoyou Li et al. This is an open access article distributed under the Creative Commons Attribution License, which permits unrestricted use, distribution, and reproduction in any medium, provided the original work is properly cited.

\begin{abstract}
We present a multiuser cooperative transmission scheme with opportunistic network coding (CTONC), which can improve system performance. In contrast to direct transmission and conventional cooperative transmission, the CTONC allows the relay node to decide whether or not to help do cooperation and employ network coding based on the limited feedback from the destinations. It will not help a transmission pair unless its direct transmission fails. This enables CTONC to make efficient use of the degrees of freedom of the channels. We derive and analyze the diversity-multiplexing tradeoff for the CTONC over Rayleigh fading channels at finite signalto-noise ratios (SNRs). Theoretical analysis and numerical results show that the proposed scheme achieves better performance gain in terms of average mutual information, outage probability, and finite-SNR diversity-multiplexing tradeoff.
\end{abstract}

\section{Introduction}

Cooperative transmission has recently received significant attention, both from academia and industry, as a new approach to achieve spatial diversity gains, increase coverage [1], and improve reliability of energy-constrained mobile devices in wireless cellular, ad hoc and sensor networks $[2,3]$. Cooperative diversity has also been widely accepted as one special effective way to combat fading over wireless channels without additional complexity of multiple-input multiple-output (MIMO) systems. In [2], the authors develop and analyze several cooperative protocols like the amplify-and-forward (AF), decode-and-forward $(\mathrm{DF})$, and their selective and incremental variations. The diversity-multiplexing tradeoff formulation is first proposed by Zheng and Tse at asymptotically high SNR [4], which is a beneficial tool to investigate the role of code design on extracting the available diversity gains 
and spatial multiplexing gains of cooperative relay systems $[5,6]$. The opportunistic relaying cooperation scheme can achieve the same diversity-multiplexing tradeoff as achieved by the distributed space-time coded cooperation scheme [7]. Narasimhan in [8] presents new definitions of diversity gain and multiplexing gain at finite SNR, due to that some practical communication systems would rather operate in low-to-moderate SNR region. In [9], the authors analyze the diversity-multiplexing tradeoff for three practical DF half-duplex cooperative protocols in a fading relay channel at finite SNRs. Liu et al. in [10] investigate the finite-SNR diversity-multiplexing tradeoff performance for the AF and DF protocols assisted by multiple relays and compare the relative outage performance of AF and DF. In this paper, we focus on the finite-SNR diversity-multiplexing tradeoff characterization for cooperative transmissions.

Recently, there have been some studies incorporating network coding [11] in cooperative communications. Network coding is a promising approach to improve spectral efficiency and reduce time-slot overhead for multiuser cooperative communications [12, 13]. Chen et al. investigate the diversity gain offered at high signal-to-noise ratios by implementing network coding at relaying nodes. In the case of user cooperation, network coding yields better diversity performance, especially when there are multiple network users in [14]. The reference [15] proposes a network-coded cooperation scheme with dynamic coding mechanism based on the observed instantaneous source-to-relay channel quality, which achieves a diversity-multiplexing tradeoff superior to conventional cooperation. In [16], the authors study the analog network coding (ANC) with AF protocol for frequency flat Rayleigh fading channels, derive a tight lower bound of outage probability in closed-form, and obtain the finite-SNR diversity-multiplexing tradeoff. However, the disadvantage of ANC scheme is noise propagation for AF relay pattern $[13,17]$. The challenging problem is, from theoretical and practical point of view, how to quantify the benefits that a wireless network can enjoy by combining network coding with cooperative transmissions, or, how much performance gain can be obtained by network-coded cooperation? To the best of our knowledge, this problem is still open. Therefore, it naturally inspires our motivation.

In this paper, we consider a cooperative network with two sources, one relay and two destinations, and study the relay cooperation assuming DF cooperation as it offers the design flexibility for implementing network coding. In such system, each node is equipped with one antenna, and the finite-SNR diversity-multiplexing tradeoff is achieved across multiple nodes via the cooperation with opportunistic network coding. "Opportunistic" means that the relay decides whether or not to help do cooperation for the $s-d$ pairs based on the limited feedbacks from the destinations (see Figure 1). It will not help forward information for the $s-d$ pair unless the feedback indicates that its direct communication fails. The advantage is that CTONC scheme allows the encoding relay node to decide whether it employs network coding based on the limited feedbacks.

The rest of this paper is organized as follows. Section 2 describes the system model. In Section 3, the CTONC scheme is presented over Rayleigh fading channels. We derive the finite-SNR diversity-multiplexing tradeoff for the CTONC in Section 4. Numerical results are provided in Section 5, showing finite-SNR diversity-multiplexing performance, average mutual information, and outage probability. Section 6 concludes the paper.

\section{System Model}

We consider a cooperative network with two sources, one relay and two destinations (see Figure 1). 


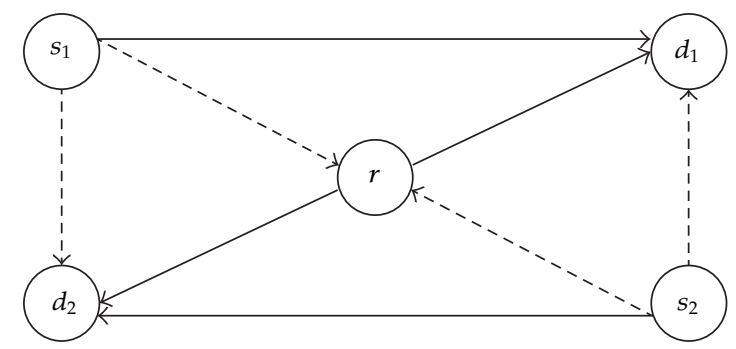

Figure 1: Multiuser cooperative transmission scenario.

The source node $s_{1}$ wants to transmit data $x_{1}$ to the destination $d_{1}$, which is overheard by the relays $r$ and $d_{1}$. And the source node $s_{2}$ wants to transmit data $x_{2}$ to the destination $d_{2}$, which is overheard by the relay $r$ and the destination $d_{1}$. In the multicast case, the node $s_{1}\left(s_{2}\right)$ wants to transmit multicast data $x_{1}\left(x_{2}\right)$ to the destinations $d_{1}, d_{2}$, and $r$. The nodes $d_{1}$ and $d_{2}$ may utilize resources more efficiently. However, the multicasting is not reliable in most wireless networks. For instance, the multicasting is lack of acknowledgments in 802.11 networks, as a result, which does not ensure a complete, reliable flow of data and may experience lower quality of service. Therefore, we only consider the former case in this paper. We assume that all nodes operate in the half-duplex mode. The multiuser cooperative transmissions are based on a synchronous time-division multiplexing. While the channel gain remains constant over one timeslot, it varies independently from one timeslot to its next. Each time slot consists of an equal amount of time. For medium access, all the transmissions happen orthogonally. We focus on orthogonal transmissions by allocating nonoverlapping time slots to different transmissions throughout the paper. All channels are assumed to be flat Rayleigh-fading, and perfect channel state information (CSI) is known at the receivers. To be specific, the received signal of node $v$ from node $u$ is given by

$$
y_{v}=h_{u v} x_{u}+z_{v}
$$

where $h_{u v}$ denotes the channel gain between transmitting node $u$ and receiving node $v$, which captures the effects of path-loss, shadowing, and frequency nonselective fading. We model $h_{u v}$ as zero-mean, independent complex Gaussian random variable with variance $\sigma_{u v}^{2}$, so that the magnitude $\left|h_{u v}\right|$ is Rayleigh distributed $\left(\left|h_{u v}\right|^{2}\right.$ is exponentially distributed with mean $\left.\sigma_{u v}^{2}\right) \cdot x_{u}$ is the transmit signal of the node $u$, and $z_{v}$ is modeled as a complex additive white Gaussian noise (AWGN) with zero-mean and variance $N_{0}$. The random noise in the form of Gaussian white noise is possibly not satisfactory but appears fluctuations of its statistical properties and exhibits randomness in the form of fractional Gaussian noise [18]. Fractional Gaussian noise is widely used in various fields, ranging from geosciences to telecommunications, which is also modeled for communication channels and internet communication $[19,20]$. It will be as our future work.

For simplicity, we assume all the transmit nodes have a common transmit power, which is denoted as $P$. The transmit SNR is denoted as $\rho$, and then we have $\rho=P / N_{0}$. With loss of generality, all nodes are assumed to transmit their data towards their destinations at a common rate of $R$ bits per channel use (bit/s/Hz). Due to the nature of fading, the receivers cannot correctly decode all the data from the transmitting nodes. We denote $I$ as the channel average mutual information, which is a function of the fading coefficients of the channel. 
The event $I<R$ is referred to as an outage event. The probability of an outage event $P(I<R)$ is referred to as the outage probability of the channel.

\section{CTONC Scheme}

In this section, we describe the CTONC scheme that exploits limited feedbacks from the destinations, for example, a single bit indicating the success or failure of the direct transmission, which can remarkably improve spectral efficiency. The proposed scheme is described in the wireless cooperative network in details as follow.

During the first and second time slots, the node $s_{1}$ and node $s_{2}$ transmit their information towards their destinations at the rate $R$. Due to the broadcast nature of wireless medium, the destination $d_{2}\left(d_{1}\right)$ can hear $x_{1}\left(x_{2}\right)$, and the relay $r$ can hear $x_{1}$ and $x_{2}$. We assume the links $s_{1} \rightarrow d_{2}, s_{2} \rightarrow d_{1}$ and $s_{i} \rightarrow r$ have their channel gains good enough to support data transmission without error. Each destination indicates the success or failure of the corresponding direct transmission by broadcasting a single bit of feedback to the source and relay, which is assumed to be reliably observed by at least the relay $r$. If the SNR between the source $s_{i}$ and destination $d_{i}$ is sufficiently high to guarantee successful direct transmission, the feedback indicates success of the direct transmission. Otherwise, the destination $d_{i}$ will send a feedback informing $r$ of the failure of its direct transmission.

The relay takes different actions based on different status of the direct transmissions of the two $s-d$ pairs. If the direct transmissions of both pairs succeed, the relay will do nothing. For direct transmission, the channel is modeled as

$$
y_{d_{i}}=h_{s_{i} d_{i}} x_{i}+z_{d_{i}}, \quad i=1,2
$$

If the direct transmission of only one of the two pairs succeeds, during the next coming time slot, the relay will decode, reencode, and forward what it receives from the source of the pair whose direct transmission fails towards the corresponding destination. For cooperative diversity transmission, after the source $s_{i}$ transmits the data $x_{i}$, the signals received at the destination $d_{i}$ and relay $r$ are modeled by

$$
\begin{gathered}
y_{r}=h_{s_{i}} x_{i}+z_{r}, \quad i=1 \text { or } 2 \\
y_{d_{i}}=h_{s_{i} d_{i}} x_{i}+z_{d_{i}}, \quad i=1 \text { or } 2 .
\end{gathered}
$$

In the next time slot for the relay transmission, the received signal at the destination $d_{i}$ is modeled as

$$
y_{d_{i}}=h_{r d_{i}} x_{r}+z_{d_{i}}
$$

After receiving the message transmitted by the sources, the relay then retransmits the same codeword in the next time slot. This constraint allows for the receiver to perform maximum ratio combining (MRC) to combine the received signals before decoding.

If the direct transmissions of both pairs fail, the relay will perform network coding (XOR operation) on the data it receives from both $s_{1}$ and $s_{2}$ before forwarding (broadcasting) 
the network-coded data towards destinations $d_{1}$ and $d_{2}$. The received signal at the relay $r$ is modeled by

$$
y_{r}=h_{s_{i} r} x_{i}+z_{r}, \quad i=1,2
$$

During the next coming time slot the relay forwards $x_{\mathrm{XOR}}=x_{1} \oplus x_{2}$ towards the two destinations. Each destination node extracts its desired data by applying XOR operation to the overhead data from the combined data. We constrain the network-coded transmit scheme to be orthogonal, so that the transmissions of the source and relay are emitted and received over parallel channels. The two parallel channels are one from the source and the other from the relay to the destination. They are modeled as, respectively,

$$
\begin{gathered}
y_{d_{i}}=h_{s_{i} d_{i}} x_{i}+z_{d_{i}} \\
y_{d_{i}}=h_{r d_{i}} x_{\mathrm{XOR}}+z_{d_{i}} .
\end{gathered}
$$

Since $d_{1}$ and $d_{2}$ have already obtained a copy of $x_{2}$ and $x_{1}$ from $s_{2}$ and $s_{1}$ by overhearing, respectively, $d_{1}$ can recover $x_{1}$ from $x_{1}=x_{2} \oplus x_{\mathrm{XOR}}=x_{2} \oplus\left(x_{1} \oplus x_{2}\right)$. Similarly, the $x_{2}$ can be retrieved at $d_{2}$. Clearly, with the help of network coding, the relay is used for cooperation for both $s-d$ pairs simultaneously. The data are aggregated and combined by network coding at the relay node and can be extracted at the destination nodes.

\section{Finite-SNR Diversity-Multiplexing Tradeoff}

In this section, we investigate the diversity-multiplexing tradeoff of the CTONC scheme over Rayleigh fading channels. While the conventional definitions of diversity and multiplexing gains of a system refer to asymptotic quantities as the SNR approaches infinity [4], here we consider the diversity and multiplexing definitions of [8] that extend these tradeoffs to finiteSNRs. The multiplexing gain $r$ is defined as the ratio of the transmission data rate $R$ to the capacity of an AWGN channel at $\operatorname{SNR} \rho$ :

$$
r=\frac{R}{\log _{2}(1+\rho)} .
$$

This definition provides an indication of the sensitivity of a rate adaptation strategy as the SNR changes. Additionally, the diversity gain $d(r, \rho)$ of a system with multiplexing gain $r$ at SN $\rho$ is defined by the negative slope of the log-log plot of outage probability versus SNR:

$$
d(r, \rho)=-\frac{\rho}{P_{\text {out }}(r, \rho)} \frac{\partial P_{\text {out }}(r, \rho)}{\partial \rho}
$$

where $P_{\text {out }}(r, \rho)$ is the outage probability as function of the multiplexing gain and SNR, which is

$$
P_{\text {out }}(r, \rho)=P\left[I<r \log _{2}(1+\rho)\right] \text {. }
$$


The finite-SNR diversity gain provides an estimate of the additional SNR required to decrease the outage probability by a specified amount for a given multiplexing gain.

We denote $I_{i}^{D}$ as the mutual information of the direct link from $s_{i}$ to $d_{i}$, and then

$$
I_{i}^{D}=\log _{2}\left(1+\rho\left|h_{s_{i}} d_{i}\right|^{2}\right), \quad i=1,2
$$

If only the direct transmission of $s-d$ pair $i(i=1$ or 2$)$ fails, the relay will take repetition-based decode-and-forward action for this pair. In the case of repetition coding at the relay, the mutual information between the source and destination of pair $i$ can be readily shown as

$$
I_{i}^{\text {noNC }}= \begin{cases}\log _{2}\left(1+\rho\left|h_{s_{i} d_{i}}\right|^{2}+\rho\left|h_{r d_{i}}\right|^{2}\right), & \text { when } \log _{2}\left(1+\rho\left|h_{s_{i} r}\right|^{2}\right)>R \\ \log _{2}\left(1+\rho\left|h_{s_{i} d_{i}}\right|^{2}\right), & \text { otherwise. }\end{cases}
$$

In the case, two pairs of direct transmissions fail, the relay will perform cooperative transmission with network coding. The transmissions, from the source to the destination and from the cooperative relay to the destination, can be viewed as parallel channels $[2,9]$. The mutual information between the source and destination of pair $i(i=1,2)$ is given as

$$
I_{i}^{\mathrm{NC}}= \begin{cases}\log _{2}\left(1+\rho\left|h_{s_{i} d_{i}}\right|^{2}\right)+\log _{2}\left(1+\rho\left|h_{r d_{i}}\right|^{2}\right), & \text { when } \log _{2}\left(1+\rho\left|h_{s_{i} r}\right|^{2}\right)>R, i=1,2 \\ \log _{2}\left(1+\rho\left|h_{s_{i} d_{i}}\right|^{2}\right), & \text { otherwise. }\end{cases}
$$

To obtain diversity-multiplexing gain tradeoff for CTONC at finite SNR, we need to compute the probability of outage and its derivative. We consider $s-d$ pair $i$ suffers an outage when $x_{i}$ cannot be correctly received at $d_{i}$. Based on (4.4)-(4.6), the probability of outage for $s_{1}-d_{1}$ pair is given by

$$
\begin{aligned}
p_{1}^{\mathrm{ONC}}= & P\left(I_{1}^{\mathrm{NC}}<R, I_{1}^{D}<R, I_{2}^{D}<R\right)+P\left(I_{1}^{\mathrm{noNC}}<R, I_{1}^{D}<R, I_{2}^{D} \geq R\right) \\
= & P\left[\log _{2}\left(1+\rho\left|h_{s_{1} d_{1}}\right|^{2}\right)+\log _{2}\left(1+\rho\left|h_{r d_{1}}\right|^{2}\right)<R\right] P\left[\log _{2}\left(1+\rho\left|h_{s_{2} d_{2}}\right|^{2}\right)<R\right] \\
& \times P\left[\log _{2}\left(1+\rho\left|h_{s_{1} r}\right|^{2}\right) \geq R\right] P\left[\log _{2}\left(1+\rho\left|h_{s_{2} r}\right|^{2}\right) \geq R\right] \\
& +P\left[\log _{2}\left(1+\rho\left|h_{s_{1} d_{1}}\right|^{2}+\rho\left|h_{r d_{1}}\right|^{2}\right)<R\right] P\left[\log _{2}\left(1+\rho\left|h_{s_{2} d_{2}}\right|^{2}\right) \geq R\right] \\
& \times P\left[\log _{2}\left(1+\rho\left|h_{s_{1} r}\right|^{2}\right) \geq R\right] .
\end{aligned}
$$


For $s_{2}-d_{2}$ pair, it is similar. Here, we define the events $A_{1}, A_{2}, A_{3}, A_{4}, A_{5}$, and $A_{6}$ as follows:

$$
\begin{aligned}
& A_{1}: \log _{2}\left(1+\rho\left|h_{s_{1} r}\right|^{2}\right) \geq R \\
& A_{2}: \log _{2}\left(1+\rho\left|h_{s_{2} r}\right|^{2}\right) \geq R \\
& A_{3}: \log _{2}\left(1+\rho\left|h_{s_{2} d_{2}}\right|^{2}\right) \geq R \\
& A_{4}: \log _{2}\left(1+\rho\left|h_{s_{2} d_{2}}\right|^{2}\right)<R \\
& A_{5}: \log _{2}\left(1+\rho\left|h_{s_{1} d_{1}}\right|^{2}+\rho\left|h_{r d_{1}}\right|^{2}\right)<R \\
& A_{6}: \log _{2}\left(1+\rho\left|h_{s_{1} d_{1}}\right|^{2}\right)+\log _{2}\left(1+\rho\left|h_{r d_{1}}\right|^{2}\right)<R .
\end{aligned}
$$

Thus, the probability of outage for $s_{1}-d_{1}$ pair is given as

$$
P_{1}^{\mathrm{ONC}}=P\left(A_{6}\right) P\left(A_{4}\right) P\left(A_{1}\right) P\left(A_{2}\right)+P\left(A_{5}\right) P\left(A_{3}\right) P\left(A_{1}\right)
$$

Additionally, the derivative of the outage probability with respect to the SNR $\rho$ is given as follows:

$$
\begin{aligned}
\frac{\partial P_{1}^{\mathrm{ONC}}}{\partial \rho}= & \frac{\partial P\left(A_{1}\right)}{\partial \rho}\left[P\left(A_{6}\right) P\left(A_{4}\right) P\left(A_{2}\right)+P\left(A_{5}\right) P\left(A_{3}\right)\right] \\
& +P\left(A_{1}\right)\left[\frac{\partial P\left(A_{6}\right)}{\partial \rho} P\left(A_{2}\right) P\left(A_{4}\right)+\frac{\partial P\left(A_{4}\right)}{\partial \rho} P\left(A_{2}\right) P\left(A_{6}\right)+\frac{\partial P\left(A_{2}\right)}{\partial \rho} P\left(A_{6}\right) P\left(A_{4}\right)\right] \\
& +P\left(A_{1}\right)\left[\frac{\partial P\left(A_{5}\right)}{\partial \rho} P\left(A_{3}\right)+\frac{\partial P\left(A_{3}\right)}{\partial \rho} P\left(A_{5}\right)\right]
\end{aligned}
$$

Substituting (4.9) and (4.10) into (4.2), we can obtain the diversity gain $d(r, \rho)$ for $s_{1}-d_{1}$ pair. Finally, what remains is to compute these probabilities and derivatives. Expect for $P\left(A_{6}\right)$, they can be derived in closed-form (see the Appendix). We focus on the computation of outage probability $P\left(A_{6}\right)$, which unfortunately cannot be computed in closed form. Instead, it is turned to the bounding technique described in $[9,21]$. Note that $R$ depends on $\rho$ via (4.1):

$$
2^{R}=(1+\rho)^{r} .
$$


For the $P\left(A_{6}\right)$, there is

$$
\begin{aligned}
P\left(A_{6}\right) & =P\left[\log _{2}\left(1+\rho\left|h_{s_{1} d_{1}}\right|^{2}\right)+\log _{2}\left(1+\rho\left|h_{r d_{1}}\right|^{2}\right)<R\right] \\
& =P\left[\left(1+\rho\left|h_{s_{1} d_{1}}\right|^{2}\right)\left(1+\rho\left|h_{r d_{1}}\right|^{2}\right)<(1+\rho)^{r}\right] \\
& \geq P\left[\left(1+\rho\left|h_{s_{1} d_{1}}\right|^{2}\right)<(1+\rho)^{a_{1}} \cap\left(1+\rho\left|h_{r d_{1}}\right|^{2}\right)<(1+\rho)^{a_{2}}\right] \\
& =P\left[\left(1+\rho\left|h_{s_{1} d_{1}}\right|^{2}\right)<(1+\rho)^{a_{1}}\right] P\left[\left(1+\rho\left|h_{r d_{1}}\right|^{2}\right)<(1+\rho)^{a_{2}}\right] \\
& =\left(1-\exp \left(-\frac{(1+\rho)^{a_{1}}-1}{\lambda_{3} \rho}\right)\right)\left(1-\exp \left(-\frac{(1+\rho)^{a_{2}}-1}{\lambda_{4} \rho}\right)\right),
\end{aligned}
$$

where $(1+\rho)^{r}=(1+\rho)^{a_{1}}(1+\rho)^{a_{2}} \cdot \lambda_{3} \rho=\sigma_{s_{1} d_{1}}^{2} \rho=\gamma_{s_{1} d_{1}}$ is the mean source-destination SNR, and $\lambda_{4} \rho=\sigma_{r d_{1}}^{2} \rho=\gamma_{r d_{1}}$ is the mean relay destination. To obtain accurate diversity gains at finite SNRs, the lower bound in (4.12) is maximized over the exponents $a_{1}$ and $a_{2}$ for each $\rho$. A feasible point for this optimization is determined by the fact that $a_{1} \geq 0$ and $a_{2} \geq 0$. This yields the following conditions:

$$
\begin{aligned}
& 0 \leq a_{1} \leq r \\
& 0 \leq a_{2} \leq r \\
& a_{1}+a_{2}=r .
\end{aligned}
$$

Using efficient nonlinear programming techniques, the computational time of $a_{1}$ and $a_{2}$ is much smaller than Monte Carlo simulations for the exact outage probability [21]. Once the optimals $a_{1}$ and $a_{2}$ are found, the outage probability and its derivative can be computed. The derivative of the bound on $P\left(A_{6}\right)$ in (4.12) with respect to $\rho$ is given by

$$
\begin{aligned}
\frac{\partial P\left(A_{6}\right)}{\partial \rho}= & \exp \left(-\frac{(1+\rho)^{a_{1}}-1}{\lambda_{3} \rho}\right)\left(\frac{-a_{1}(1+\rho)^{a_{1}-1}}{\lambda_{3} \rho}+\frac{\lambda_{3}\left((1+\rho)^{a_{1}}-1\right)}{\left(\lambda_{3} \rho\right)^{2}}\right) \\
& \times\left(1-\exp \left(-\frac{(1+\rho)^{a_{2}}-1}{\lambda_{4} \rho}\right)\right)+\left(1-\exp \left(-\frac{(1+\rho)^{a_{1}}-1}{\lambda_{3} \rho}\right)\right) \\
& \times \exp \left(-\frac{(1+\rho)^{a_{2}}-1}{\lambda_{4} \rho}\right)\left(\frac{-a_{2}(1+\rho)^{a_{2}-1}}{\lambda_{4} \rho}+\frac{\lambda_{4}\left((1+\rho)^{a_{2}}-1\right)}{\left(\lambda_{4} \rho\right)^{2}}\right) .
\end{aligned}
$$

\section{Numerical Results}

In this section, we demonstrate the performance of the CTONC scheme. For the simplicity, we assume that the pairs $s_{1}-d_{1}$ and $s_{2}-d_{2}$ are symmetrical; that is, they have the same power and channel gains. This section provides some numerical results on mutual information, outage probability, and diversity-multiplexing tradeoff for the pair $s_{1}-d_{1}$. For the pair $s_{2}-d_{2}$, 


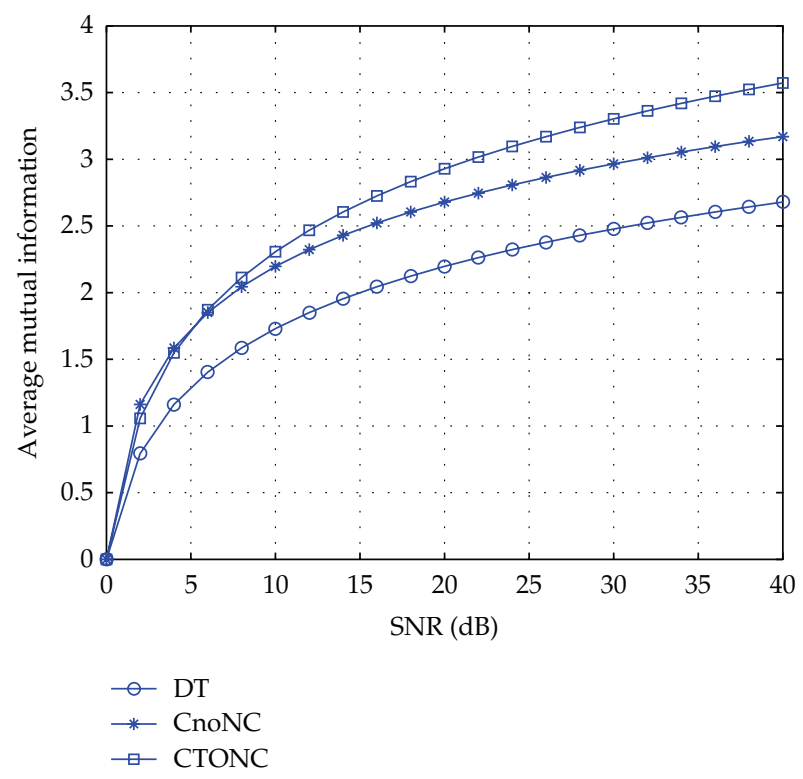

Figure 2: Mutual information comparison of the three schemes.

it is similar. The performance comparison of direct transmission scheme (DT), cooperative non-NC transmission scheme (CnoNC), and CTONC scheme is presented under different conditions as follows.

Figure 2 depicts the mutual information versus SNR for different schemes. We can see that both CTONC and CnoNC schemes increase the mutual information. However, the mutual information of CnoNC scheme is smaller than that of CTONC scheme when SNR $\rho>6$. The outage probabilities versus transmission rate under all three schemes are illustrated in Figure 3. It is shown that the outage probability of CTONC scheme is smaller than that of other schemes. The performance of CnoNC scheme is superior to DT scheme. As the transmission rate increases, the outage probability of the CTONC scheme increases more slowly than other schemes, meaning that the proposed scheme is less sensitive to the transmission rate variations compared with the direct transmission and cooperative transmission without network coding.

In Figure 4, we compare the finite-SNR diversity-multiplexing tradeoff performance of the three schemes at SNR values of 0 and $40 \mathrm{~dB}$. As the multiplexing gains increase, the diversity gains degrade. The DT and CTONC schemes achieve the multiplexing gain of upper bound. The DT scheme can achieve larger diversity gains than CTONC scheme for high multiplexing gains at low SNR. However, the diversity gain of the CTONC and CnoNC schemes are superior to DT scheme for low multiplexing gains. The maximum achievable multiplexing gain of the CnoNC scheme is 0.5 .

The outage probability versus SNR for the three schemes is illustrated at different multiplexing gains in Figure 5. We can see that the outage probability performance of CTONC scheme is optimal at multiplexing gain $r=0.25$ and is better than that of other schemes for high SNR at multiplexing gain $r=0.5$. In addition, the outage probability performance of CTONC and CnoNC schemes is always superior to the DT schemes across the whole range of SNR and at different multiplexing. Furthermore, as the multiplexing gain increases, it is clearly shown that the outage probability performance of all the three schemes degrades. 


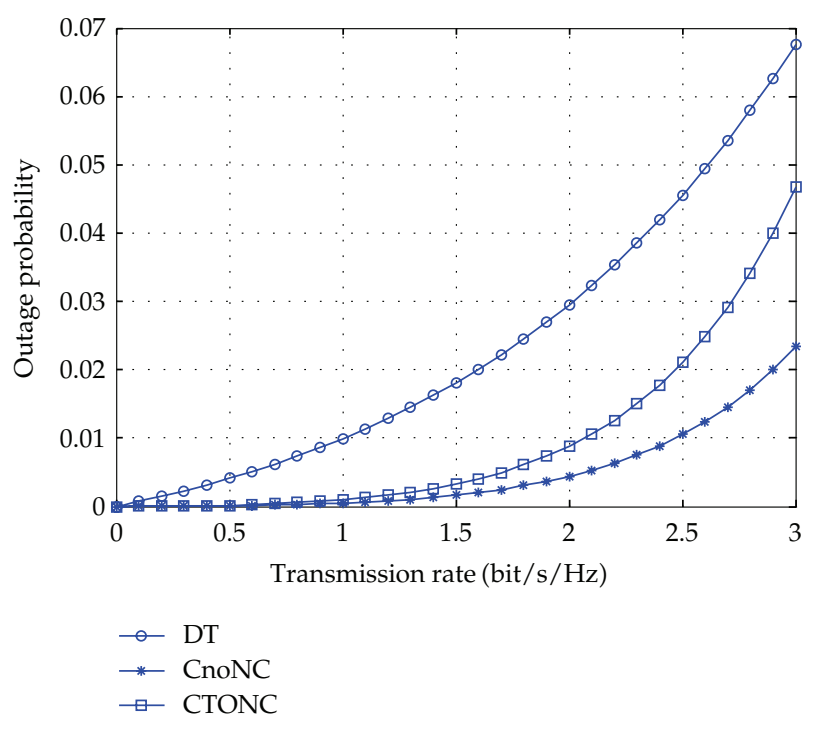

Figure 3: Outage probability comparison of the three schemes.

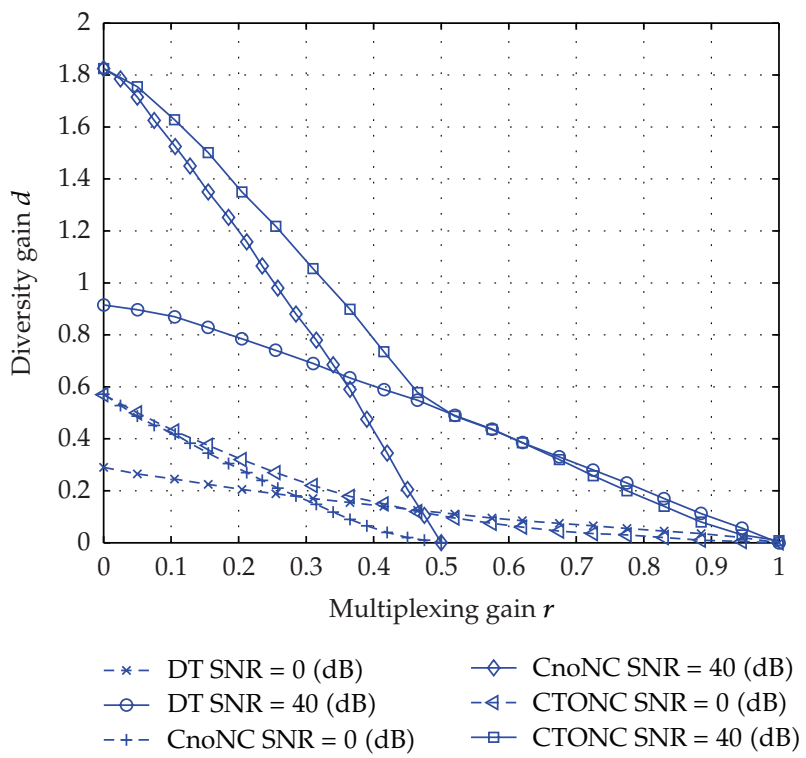

Figure 4: Finite-SNR diversity-multiplexing comparison of the three schemes.

\section{Conclusion}

In this paper, we propose the opportunistic network coding for relay cooperative transmissions to provide a feasible method for performance improvement and study mutual information, outage probability, and the finite-SNR diversity-multiplexing tradeoff. On the whole, the proposed CTONC scheme outperforms other schemes in terms of mutual information and outage probability. For low multiplexing gains, the CTONC scheme achieves larger diversity gains than other schemes at finite SNRs. As a future work, we will extend the proposed scheme to cooperative MIMO in the context of cellular mobile systems, consider fractional Gaussian noise and further investigate performance gains. 


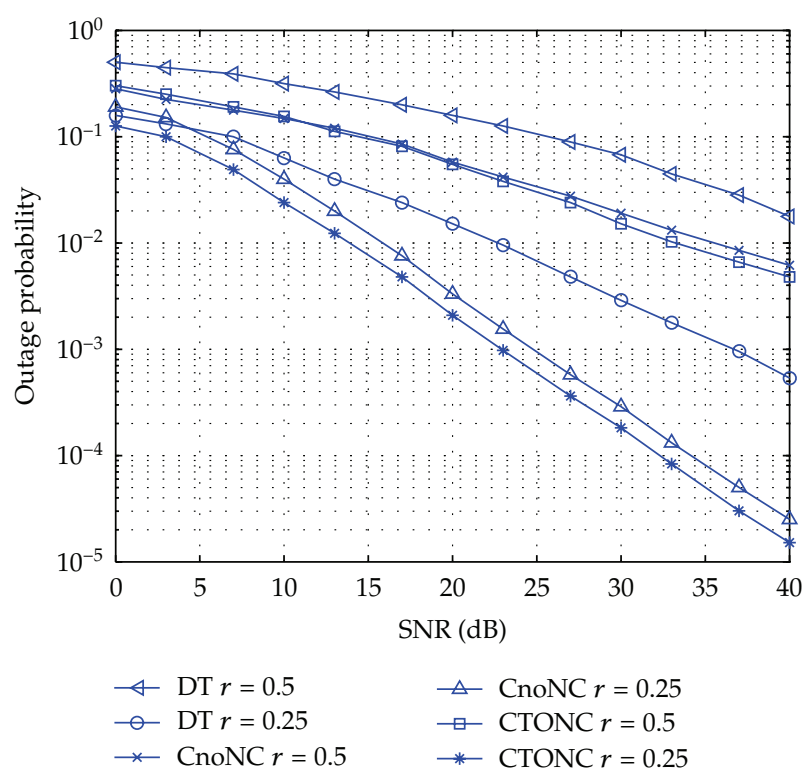

Figure 5: Outage probability comparison of the three schemes with different multiplexing gains.

\section{Appendix}

\section{Outage Probability Derivation}

Obviously, $\left|h_{u v}\right|^{2}$ is exponentially distributed with parameter $\sigma_{u v}^{-2}$. Let $X=\rho\left|h_{u v}\right|^{2}$ be exponential random variable with parameter $1 / \lambda \rho$, where $\lambda=\sigma_{u v}^{2}$. Thus, the PDF of $X$ can be expressed as

$$
p_{x}(x)=\frac{1}{\lambda \rho} \exp \left(-\frac{x}{\lambda \rho}\right) \quad x \geq 0
$$

Using the above PDF, the outage probability is given as follows:

$$
\begin{aligned}
P\left(A_{1}\right) & =P\left[\log _{2}\left(1+\rho\left|h_{s_{1} r}\right|^{2}\right) \geq R\right] \\
& =1-P\left[\left(\rho\left|h_{s_{1} r}\right|^{2}\right)<2^{R}-1\right] \\
& =1-\int_{0}^{2^{R}-1} p_{x}(x) d x \\
& =\exp \left(-\frac{2^{R}-1}{\lambda_{1} \rho}\right) \\
& =\exp \left(-\frac{(1+\rho)^{r}-1}{\lambda_{1} \rho}\right),
\end{aligned}
$$


where $\lambda_{1}=\sigma_{s_{1} r}^{2}$. Then, the derivative $\partial P\left(A_{1}\right) / \partial \rho$ can be calculated by

$$
\frac{\partial P\left(A_{1}\right)}{\partial \rho}=\exp \left(-\frac{(1+\rho)^{r}-1}{\lambda_{1} \rho}\right)\left(\frac{-r(1+\rho)^{r-1}}{\lambda_{1} \rho}+\frac{\lambda_{1}\left((1+\rho)^{r}-1\right)}{\left(\lambda_{1} \rho\right)^{2}}\right) .
$$

Based on (4.8), (A.2), and (A.3), for $\lambda_{2}=\sigma_{s_{2} r}^{2}$ and $\lambda_{5}=\sigma_{s_{2} d_{2}}^{2}$, we can easily obtain $P\left(A_{2}\right)$, $\partial P\left(A_{2}\right) / \partial \rho, P\left(A_{3}\right)$, and $\partial P\left(A_{3}\right) / \partial \rho$, respectively.

Similarly, the probability of outage $P\left(A_{4}\right)$ and its derivative are given by

$$
\begin{gathered}
P\left(A_{4}\right)=P\left[\log _{2}\left(1+\rho\left|h_{s_{2} d_{2}}\right|^{2}\right)<R\right] \\
=1-\exp \left(-\frac{(1+\rho)^{r}-1}{\lambda_{5} \rho}\right) \\
\frac{\partial P\left(A_{4}\right)}{\partial \rho}=-\exp \left(-\frac{(1+\rho)^{r}-1}{\lambda_{5} \rho}\right)\left(\frac{-r(1+\rho)^{r-1}}{\lambda_{5} \rho}+\frac{\lambda_{5}\left((1+\rho)^{r}-1\right)}{\left(\lambda_{5} \rho\right)^{2}}\right) .
\end{gathered}
$$

The $P\left(A_{2}\right)$ is shown as

$$
\begin{aligned}
P\left(A_{5}\right) & =P\left[\log _{2}\left(1+\rho\left|h_{s_{1} d_{1}}\right|^{2}+\rho\left|h_{r d_{1}}\right|^{2}\right)<R\right] \\
& = \begin{cases}1-\left(1+\frac{(1+\rho)^{r}-1}{\lambda_{3} \rho}\right) \exp \left(-\frac{(1+\rho)^{r}-1}{\lambda_{3} \rho}\right), & \lambda_{3}=\lambda_{4} \\
1-\frac{\lambda_{3} \rho}{\lambda_{3} \rho-\lambda_{4} \rho} \exp \left(-\frac{(1+\rho)^{r}-1}{\lambda_{3} \rho}\right)-\frac{\lambda_{4} \rho}{\lambda_{4} \rho-\lambda_{3} \rho} \exp \left(-\frac{(1+\rho)^{r}-1}{\lambda_{4} \rho}\right), & \lambda_{3} \neq \lambda_{4},\end{cases}
\end{aligned}
$$

where $\lambda_{3}=\sigma_{s_{1} d_{1}}^{2}$ and $\lambda_{4}=\sigma_{r d_{1}}^{2}$. When $\lambda_{3}=\lambda_{4}$, its derivative is given below

$$
\begin{aligned}
\frac{\partial P\left(A_{5}\right)}{\partial \rho}= & -\exp \left(-\frac{(1+\rho)^{r}-1}{\lambda_{3} \rho}\right)\left(\frac{r(1+\rho)^{r-1}}{\lambda_{3} \rho}-\frac{\lambda_{3}(1+\rho)^{r}-1}{\left(\lambda_{3} \rho\right)^{2}}\right) \\
& -\left(1+\frac{(1+\rho)^{r}-1}{\lambda_{3} \rho}\right) \exp \left(-\frac{(1+\rho)^{r}-1}{\lambda_{3} \rho}\right)\left(\frac{-r(1+\rho)^{r-1}}{\lambda_{3} \rho}+\frac{\lambda_{3}\left((1+\rho)^{r}-1\right)}{\left(\lambda_{3} \rho\right)^{2}}\right) \\
= & \frac{(1+\rho)^{r}-1}{\lambda_{3} \rho} \exp \left(-\frac{(1+\rho)^{r}-1}{\lambda_{3} \rho}\right)\left(\frac{r(1+\rho)^{r-1}}{\lambda_{3} \rho}-\frac{\lambda_{3}\left((1+\rho)^{r}-1\right)}{\left(\lambda_{3} \rho\right)^{2}}\right) .
\end{aligned}
$$


For $\lambda_{3} \neq \lambda_{4}$, there is the following:

$$
\begin{aligned}
\frac{\partial P\left(A_{5}\right)}{\partial \rho}=\frac{\lambda_{3}-\lambda_{4}}{\left(\lambda_{3} \rho-\lambda_{4} \rho\right)^{2}} & {\left[\lambda_{3} \rho \exp \left(-\frac{(1+\rho)^{r}-1}{\lambda_{4} \rho}\right)-\lambda_{4} \rho \exp \left(-\frac{(1+\rho)^{r}-1}{\lambda_{4} \rho}\right)\right] } \\
-\frac{1}{\lambda_{3} \rho-\lambda_{4} \rho} & {\left[\lambda_{3} \exp \left(-\frac{(1+\rho)^{r}-1}{\lambda_{3} \rho}\right)+\lambda_{3} \rho \exp \left(-\frac{(1+\rho)^{r}-1}{\lambda_{3} \rho}\right)\right.} \\
& \left.\times\left(\frac{-r(1+\rho)^{r-1}}{\lambda_{3} \rho}+\frac{\lambda_{3}\left((1+\rho)^{r}-1\right)}{\left(\lambda_{3} \rho\right)^{2}}\right)\right] \\
+\frac{1}{\lambda_{3} \rho-\lambda_{4} \rho}\left[\lambda_{4} \exp \left(-\frac{(1+\rho)^{r}-1}{\lambda_{4} \rho}\right)\right. & \left.+\lambda_{4} \rho \exp \left(-\frac{(1+\rho)^{r}-1}{\lambda_{4} \rho}\right)\left(\frac{-r(1+\rho)^{r-1}}{\lambda_{4} \rho}+\frac{\lambda_{4}\left((1+\rho)^{r}-1\right)}{\left(\lambda_{4} \rho\right)^{2}}\right)\right] .
\end{aligned}
$$

Based on the above formulas, we can characterize the outage probability and finite-SNR diversity-multiplexing tradeoff performances.

\section{Acknowledgment}

This work has been supported by National S\&T Major Program (no. 2010ZX03003-003-01), the National Natural Science Foundation of China (no. 61070204), and the Fundamental Research Funds for the Central Universities (BUPT2010PTB0503), the Open-ended fund program of Guangxi Key Lab of Wireless Wideband Communication \& Signal Processing (no. 21106).

\section{References}

[1] A. Sendonaris, E. Erkip, and B. Aazhang, "User cooperation diversity—part I: system description," IEEE Transactions on Communications, vol. 51, no. 11, pp. 1927-1938, 2003.

[2] J. N. Laneman, D. N. C. Tse, and G. W. Wornell, "Cooperative diversity in wireless networks: efficient protocols and outage behavior," IEEE Transactions on Information Theory, vol. 50, no. 12, pp. 3062-3080, 2004.

[3] G. Kramer, I. Marić, and R. D. Yates, "Cooperative communications," Foundations and Trends in Networking, vol. 1, no. 3-4, pp. 271-425, 2006.

[4] L. Zheng and D. N. C. Tse, "Diversity and multiplexing: a fundamental tradeoff in multiple-antenna channels," IEEE Transactions on Information Theory, vol. 49, no. 5, pp. 1073-1096, 2003.

[5] K. Azarian, H. El Gamal, and P. Schniter, "On the achievable diversity-multiplexing tradeoff in halfduplex cooperative channels," IEEE Transactions on Information Theory, vol. 51, no. 12, pp. 4152-4172, 2005.

[6] A. Bletsas, A. Khisti, D. P. Reed, and A. Lippman, "A simple cooperative diversity method based on network path selection," IEEE Journal on Selected Areas in Communications, vol. 24, no. 3, pp. 659-672, 2006.

[7] S. Loyka and G. Levin, "Diversity-multiplexing tradeoff in the low-SNR regime," IEEE Communications Letters, vol. 15, no. 5, pp. 542-544, 2011. 
[8] R. Narasimhan, "Finite-SNR diversity-multiplexing tradeoff for correlated Rayleigh and Rician MIMO channels," IEEE Transactions on Information Theory, vol. 52, no. 9, pp. 3965-3979, 2006.

[9] E. Stauffer, O. Oyman, R. Narasimhan, and A. Paulraj, "Finite-SNR diversity-multiplexing tradeoffs in fading relay channels," IEEE Journal on Selected Areas in Communications, vol. 25, no. 2, pp. 245-257, 2007.

[10] Y. Liu, P. Dharmawansa, M. R. McKay, and K. B. Letaief, "Finite-SNR diversity-multiplexing trade-off of dual hop multiple-relay channels," IEEE Transaction on Communications, vol. 60, no. 5, pp. 14511463, 2012.

[11] R. Ahlswede, N. Cai, S. Y. Li, and R. Yeung, "Network information flow," IEEE Transactions on Information Theory, vol. 46, no. 4, pp. 1204-1216, 2000.

[12] S. Katti, H. S. Rahul, W. Hu, D. Katabi, M. Médard, and J. Crowcroft, "XORs in the air: practical wireless network coding," in Proceedings of the ACM SIGCOMM, pp. 243-254, 2006.

[13] S. Sharma, Y. Shi, J. Liu, Y. T. Hou, and S. Kompella, "Is network coding always good for cooperative communications?" in Proceedings of the IEEE INFOCOM 2010, pp. 1-9, San Diego, Calif, USA, March 2010.

[14] Y. Chen, S. Kishore, and J. Li, "Wireless diversity through network coding," in Proceedings of the IEEE Wireless Communications and Networking Conference (WCNC'06), vol. 3, pp. 1681-1686, April 2006.

[15] C. Peng, Q. Zhang, M. Zhao, Y. Yao, and W. Jia, "On the performance analysis of network-coded cooperation in wireless networks," IEEE Transactions on Wireless Communications, vol. 7, no. 8, pp. 3090-3097, 2008.

[16] Z. Yi, M. Ju, and I. M. Kim, “Outage probability and optimum power allocation for analog network coding," IEEE Transactions on Wireless Communications, vol. 10, no. 2, pp. 407-412, 2011.

[17] I. Maric, A. Goldsmith, and M. Medard, "Analog network coding in the high-SNR regime," in Proceedings of the IEEE Wireless Network Coding Conference (WiNC'2010), pp. 1-6, Bostn, Mass, USA.

[18] M. Li, Y. Q. Chen, J. Y. Li, and W. Zhao, "Hölder scales of sea level," Mathematical Problems in Engineering, vol. 2013, Article ID 863707, 2013.

[19] M. Li and W. Zhao, "Quantitatively investigating the locally weak stationarity of modified multifractional Gaussian noise," Physica A, vol. 391, no. 24, pp. 6268-6278, 2012.

[20] M. Li and W. Zhao, "On 1/f noise," Mathematical Problems in Engineering, vol. 2013, Article ID 673648, 2013.

[21] R. Narasimhan, "Finite-SNR diversity performance of rate-adaptive MIMO systems," in Proceedings of the IEEE Global Telecommunications Conference (GLOBECOM'05), pp. 1461-1465, December 2005. 


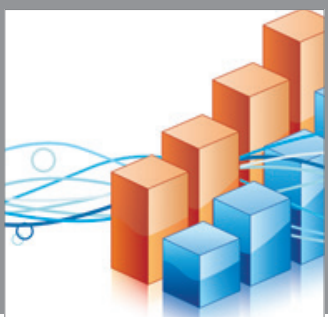

Advances in

Operations Research

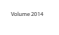

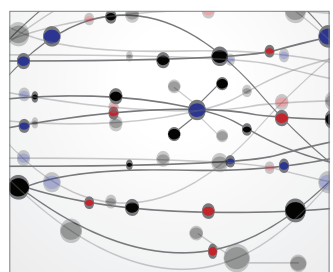

\section{The Scientific} World Journal
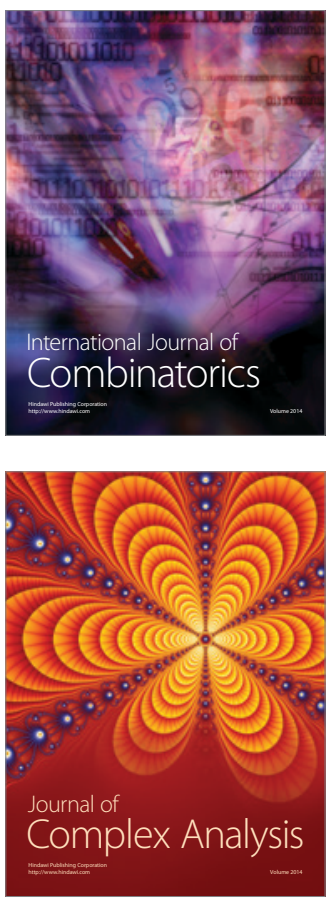

International Journal of

Mathematics and

Mathematical

Sciences
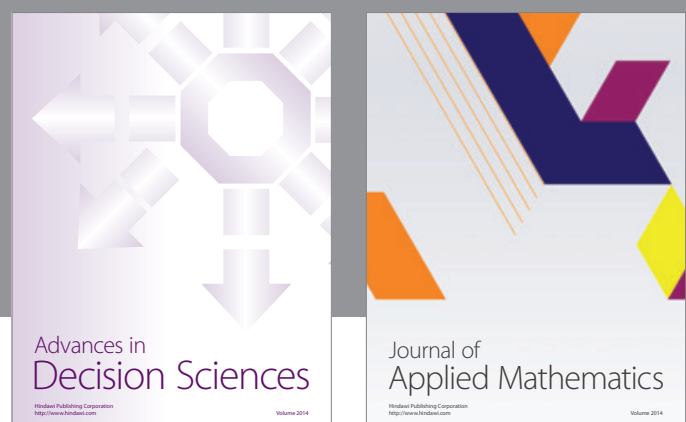

Journal of

Applied Mathematics
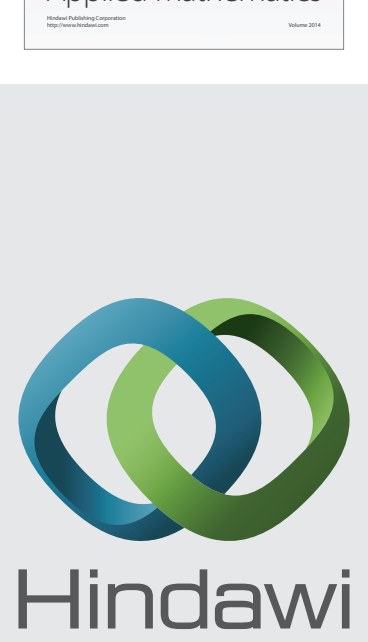

Submit your manuscripts at http://www.hindawi.com
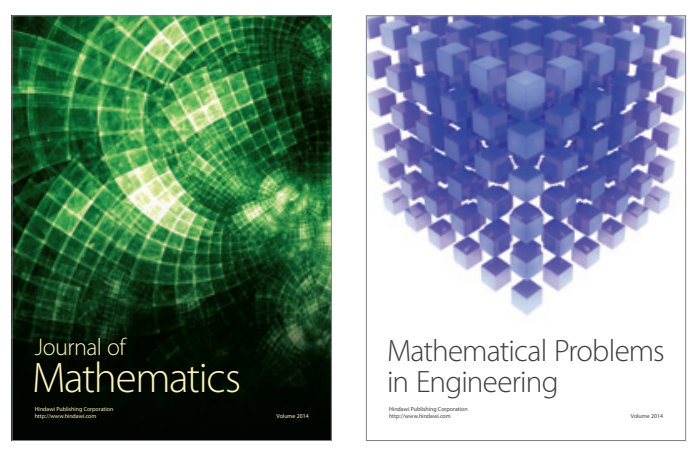

Mathematical Problems in Engineering
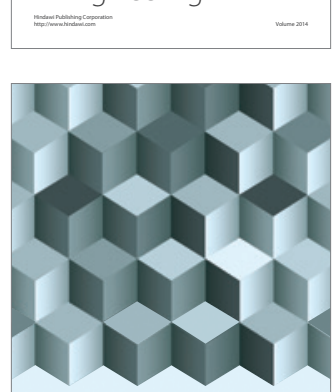

Journal of

Function Spaces
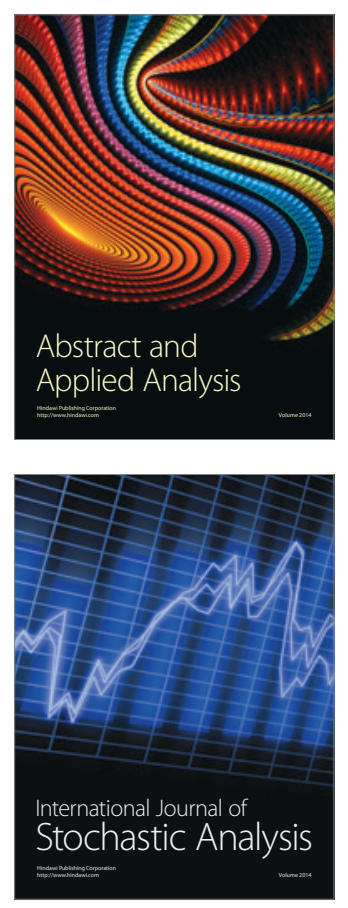

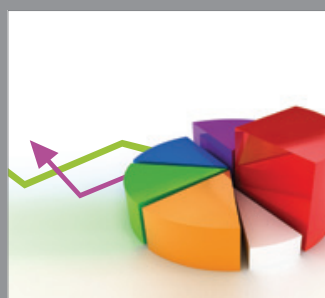

ournal of

Probability and Statistics

Promensencen
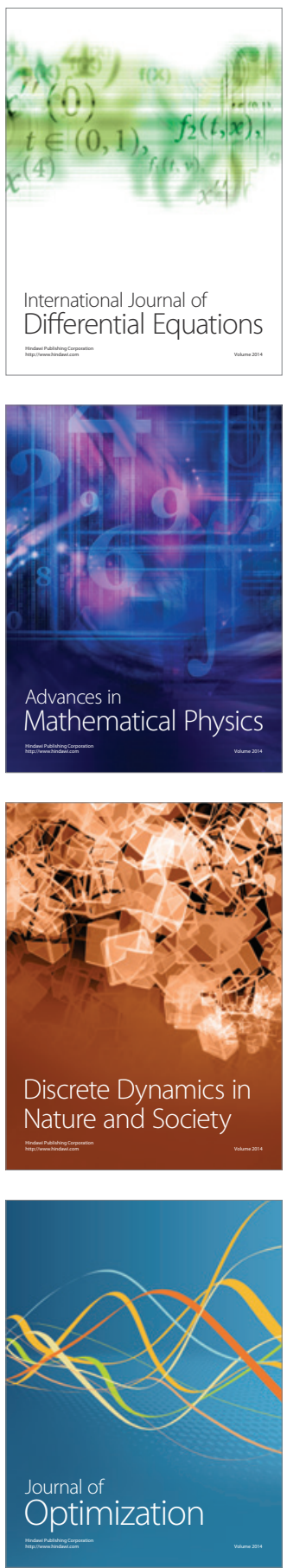